\title{
On Streamwise Vortices in Large Eddy Simulations of Initially Laminar Plane Mixing Layers
}

\author{
W. A. McMullan*, S. J. Garrett \\ Department of Engineering, University of Leicester, University Road, Leicester, LE1 \\ 7RH, United Kingdom
}

\begin{abstract}
This article details the influence of the nature of imposed inflow fluctuations on Large Eddy Simulations of a spatially developing turbulent mixing layer originating from laminar boundary layers. A simulation with imposed white-noise random fluctuations, commonly used in numerical simulations, produces mean-flow statistics that agree well with reference experimental data. Whilst flow visualisation images show evidence for streamwise vorticity in this simulation, quantitative statistics do not reveal the presence of statistically stationary streamwise vortices. A further simulation that uses physically-correlated inflow fluctuations also produces good mean-flow statistical agreement with reference data. From secondary shear stress contours it can be inferred that this simulation does, however, predict the presence of statistically stationary streamwise vortices. The properties of the streamwise vortices are in good agreement with experimental data. The data presented here indicate that, even for initially laminar conditions, plane mixing layer simulations require accurate physically correlated inflow conditions in order
\end{abstract}

\footnotetext{
*Corresponding author: andrew.mcmullan@le.ac.uk
} 
to reproduce the flow features found experimentally.

Keywords: Large Eddy Simulation, Streamwise Vortices, Mixing Layers

\section{Introduction}

Plane turbulent mixing layers have been the focus of extensive research since the 1940s. The laboratory mixing layer flow is typically composed of two parallel streams of fluid of differing velocity (and density), brought into contact at the end of a splitter plate; this is known as a two-stream mixing layer. A single-stream shear layer can also be produced from a high-speed stream emanating into quiescent fluid; this is often referred to as a half-jet flow.

The earliest research into mixing layers has shown that, in spite of their geometrical simplicity, the flow displays an extreme sensitivity to initial conditions [1] - [3]. In two-stream mixing layers, tripping of the high-speed boundary layer produces a growth rate that is reduced when compared to a mixing layer developing from initially laminar conditions [4], [5]. Even for mixing layers originating from initially laminar conditions, there exists an extremely large variation in the reported spreading rates of the flow (see Brown and Roshko [6] for a review). These experimental results suggest that the initial conditions persist for extremely long distances downstream of the splitter plate trailing edge. In order for a shear layer to attain self-similarity, a postulated streamwise distance of one thousand initial momentum high-seed side boundary layer thicknesses has gained widespread acceptance [7].

The seminal research of Brown and Roshko reported the discovery of quasi-two-dimensional, vortex-like structures embedded in the turbulent flow 
[6]. These structures occupied the entire visual thickness of the flow, and as such they were viewed as being of dynamical significance. Extensive research undertaken since their discovery has sought to understand the connection between the large-scale coherent structure dynamics, and the growth of the mixing layer [8] - [12].

Experimental work has shown that a mixing layer originating from initially laminar conditions undergoes a transition to turbulence, commonly referred to as the mixing transition [13]. The mixing transition is precipitated by a pairing interaction between primary vortices [14], when the local Reynolds number of the flow is of the order of 10,000, based on the visual thickness of the flow and its velocity difference [15]. A further requirement for the transition to occur is the formation of a secondary, streamwise vortex structure in the interconnecting braid region between the primary structures [14]. This secondary, streamwise orientated structure can manifest itself as 'streaks' in plan-view flow visualisation images [13]. Experiments have shown that, for a given set of initial conditions, these secondary structures appear to form at fixed spanwise locations [14], [16], [17] and persist far into the what would normally be considered the fully developed self-similar region [16]. Extensive experimental work has been undertaken to ascertain the origin of the streamwise vorticity and it has been found that the position of the streaks across the span can change, depending on the upstream flow conditions [17], [18], [19]. Theoretical analysis has shown that streamwise vorticity can be formed through helical pairings, or via a translative instability [20].

Complementary to experimental research, the plane mixing layer has been viewed as a canonical numerical test case for the last 30 years. The earliest 
computational studies of the mixing layer were of the temporal form, where the freestreams are set in opposition and the flow evolves temporally in a doubly-periodic box. Temporal simulations have produced valuable insights into the flow development [21] - [23], and have shown a sensitivity to the nature of the imposed inflow conditions [24]. It has also been shown that the spanwise scale of streamwise vorticity in temporal mixing layers can persist for long time periods, surviving several generations of pairings of the primary spanwise vortices [25].

Modern computing power has brought the simulation of the spatiallyevolving laboratory flow within reach. Recent Direct Numerical Simulation (DNS) studies of the mixing layer have shown that it is now possible to produce high-fidelity simulations of the flow in the post-transition region [26], [27]. Given the extreme computational challenge of DNS, it is more common for numerical studies of spatially developing mixing layers to use the Large Eddy Simulation (LES) methodology. Large Eddy Simulations of the spatially-developing mixing layer have shown that the mixing transition can be captured [28], and the flow is less sensitive to the choice of subgrid-scale model [29], [30] than its temporal counterpart [31]. It has also been shown that the reduction in growth rate caused by initially-turbulent conditions can be replicated using LES [32].

The experiments described above note that the mixing layer exhibits a hypersensitivity to its initial conditions, and the controlled environment offered by numerical simulations permits the careful investigation of the response of the mixing layer to imposed inflow conditions. In initially-laminar, spatially-developing simulations it is common practice to specify simple in- 
flow boundary conditions, normally based on either a hyperbolic tangent inflow velocity profile [26], [27], [28], [33], [35], [36], or on Blasius laminar boundary layer profiles [30], [33], [34]. It is common practice to impose some form of white noise random disturbance fluctuation environment onto the mean inflow velocity profile to account for the background turbulence environment in the flow. Inflow generation techniques which produce physically correlated inflow conditions are typically applied to initially-turbulent flows [37], [38], [39], but to the authors' knowledge no study has attempted to use an inflow generation technique to produce correlated fluctuations for a plane mixing layer originating from initially laminar conditions.

The aim of this research is to investigate the effect of the nature of imposed fluctuations on the initially-laminar mixing layer. The reference experimental data for this study comes from the experiments of Browand and Latigo [4]. Two distinct inflow conditions will be used in the simulations; the first is obtained from Blasius laminar boundary-layer profiles with superimposed white noise disturbances, and the second is an inflow condition generated by a recycling and rescaling method [37]. Statistically at least, both simulations originate from the same set of initial (inflow) conditions. The effect of the nature of the imposed disturbance environment on the development of the mixing layer will be investigated through mean statistical properties, and through numerical flow visualisation. The numerical methods used in the research code are outlined in Section 2. The simulation setup and flow parameters are outlined in Section 3. Results are presented in Section 4 , with a discussion of the results in the context of other simulation data in Section 5. Conclusions are drawn in Section 6. 


\section{Numerical Methods}

Here we provide a brief description of the code used in this study. A more complete description can be found elsewhere [28]. The research code is based on the spatially filtered equations for conservation of momentum and mass of a uniform density fluid. The equations are discretised on a staggered mesh using a finite volume approach. A second-order central-differencing scheme is used to solve the governing equations. The Wall Adapting Local Eddy-viscosity (WALE) model [40] is used to model the unresolved scales, with the eddy viscosity, $\nu_{s g}$, calculated from

$$
\nu_{s g}=\left(C_{w} \Delta\right)^{2} \frac{\left(S_{i j}^{d} S_{i j}^{d}\right)^{3 / 2}}{\left(\bar{S}_{i j} \bar{S}_{i j}\right)^{5 / 2}+\left(S_{i j}^{d} S_{i j}^{d}\right)^{5 / 4}}
$$

where $S_{i j}^{d}=\frac{1}{2}\left(\bar{g}_{i j}^{2}+\bar{g}_{j i}^{2}\right)-\frac{1}{3} \delta_{i j} \bar{g}_{k k}^{2}, \bar{g}_{i j}=\partial \bar{u}_{i} / \partial x_{j}$, and $C_{w}$ is a model constant specified a priori. The WALE model is attractive for the simulation of free shear flows with initially laminar conditions, as it predicts zero eddy viscosity in the presence of pure shear. It has been shown in other work by the author that this model produces improved plane mixing layer predictions when compared to the standard Smagorinsky model [30]. Temporal advancement of the governing equations is performed using a second-order accurate Adams-Bashforth method, and a multi-grid method is used to solve the pressure correction equation. A standard convective outflow condition is applied at the downstream end of the computational domain [41].

For the purposes of flow visualisation a passive scalar is introduced into the flow domain. The filtered scalar transport equation is closed by the commonly-used gradient-diffusion model, with the turbulent Schmidt number for the subgrid scalar flux set to $S c_{t}=0.3$. The scalar is discretised on the 


\begin{tabular}{ccccc}
$U_{1}\left(\mathrm{~ms}^{-1}\right)$ & $\theta_{1}(\mathrm{~mm})$ & $U_{2}\left(\mathrm{~ms}^{-1}\right)$ & $\theta_{2}(\mathrm{~mm})$ & $\mathrm{R}$ \\
\hline 25.6 & 0.457 & 5.2 & 0.86 & 0.66 \\
\hline
\end{tabular}

Table 1: Flow Parameters.

staggered mesh at the cell centre, and a second-order upwinding scheme is used to calculate the scalar flux between cell faces. The second-order AdamsBashforth method is used to integrate the scalar field forward in time.

Where the inflow condition for the simulation requires correlated fluctuations, the recycling and rescaling technique of Xiao et al. [37] is employed. The time-dependent inflow data is generated in a 'virtual domain' upstream of the main computational domain. The virtual domain is initialised with mean laminar boundary layer velocity profile data, onto which $0.1 U$ white noise fluctuations are superposed, where $U$ is the freestream velocity of the boundary layer flow. At each time step, the instantaneous flow field at a specified streamwise location a short distance upstream of the end of the virtual domain is recycled onto the inlet plane. At specified intervals, the velocity field within the virtual domain is globally rescaled to produce a desired set of velocity fluctuation statistics. A more complete description can be found in [37]. This generation method has been used in planar mixing layer [37], round jet [42], and liquid droplet breakup simulations [43], with good flow statistics obtained in each case. 


\section{Simulation Setup}

The mixing layer developing from laminar boundary layers reported by Browand and Latigo provides the reference data for this computational study [4]. The parameters of the experimental conditions are given in Table 2. The velocity ratio parameter, $R$, is defined as

$$
R=\frac{U_{1}-U_{2}}{U_{1}+U_{2}}
$$

where $U_{1}$ and $U_{2}$ are the high-speed and low-speed freestream velocities respectively. In the experimental facility, the upper and lower walls of the rig were fixed, resulting in an adverse pressure gradient in the test section. As such, a nominal velocity ratio parameter of the flow is calculated at the trailing edge of the splitter plate as $R=0.66$.

Two distinct simulations are performed in this study. Case LWN (Laminar White Noise) simulates a mixing layer originating from an inflow condition generated from two Blasius boundary layer profiles, onto which white noise is superimposed at each time step. Case LCF (Laminar Correlated Fluctuations) simulates a mixing layer with an inflow condition obtained from the recycling and rescaling method proposed by Xiao et al. [43]. Both simulations share the same mean and root mean squared (r.m.s.) velocity statistics at the trailing edge of the splitter plate $\left(x / \theta_{i}=0\right)$. These statistical quantities for the high-speed side boundary layer are shown in Figure 1, where the notation \langle\rangle$_{z}$ denotes a quantity that has been spanwise-averaged. It should be noted that, in the experiment, only the mean streamwise velocity and streamwise r.m.s. statistics were obtained in the boundary layer. In addition, there was a $1 \%$ error in the measurement of the streamwise velocity 
fluctuation profile. As such, it is impossible to precisely replicate the initial conditions of the experiment, and the inflow conditions reported here should be considered as representative of the conditions that may have existed in the experiment. The fluctuations reported here are similar to those in both magnitude and distribution to those found in other experiments [44], [45], and numerical simulations [46]. It should also be noted that a study of the effects of the magnitude of the imposed inflow disturbances is far beyond the scope of the research presented here; in this study the primary interest lies in the effect of the nature of the imposed fluctuations for a given set of mixing layer initial conditions.

Both simulations have a non-dimensional time step of $\Delta t /\left(\theta_{i} / U_{c}\right)=$ 0.0205. Statistical samples are obtained over a period of sixteen convective flow-through times. Spanwise averaging is performed on the flow statistics in order to increase the total number of samples. The upper and lower guide-walls in both simulations are modelled as slip walls, and the spanwise boundaries are periodic. Note that a previous study has shown that the initial development of the mixing layer is insensitive to a choice of WALE subgrid scale model parameter in the range of $C_{w}=0.3-0.56$ [30], and we have taken $C_{w}=0.56$ here. The passive scalar has a value of unity in the high-speed stream, and zero in the low-speed stream, and is used primarily in this study as a means of flow visualisation.

Details specific to the setup of each simulation are described in turn below.

\subsection{Case $L W N$}

In Case LWN, only the mixing layer is simulated, with the computational domain beginning at the trailing edge of the splitter plate. No solid geome- 
try is included within the domain. An extent of $1630 \times 1326 \times 392 \theta_{i}$ in the streamwise, cross-stream, and spanwise directions respectively is included in the domain. In the experiment it was found that the initial momentum thickness, $\theta_{i}$, was equal to the momentum thickness of the high-speed side boundary layer at the trailing edge of the splitter plate, $\theta_{i}=\theta_{1}$. This relationship is assumed in the current simulations. The momentum thickness of the mixing layer is obtained from

$$
\theta=\frac{1}{\Delta U^{2}} \int_{-\infty}^{\infty}\left(U_{1}-\left\langle\bar{u}_{t}\right\rangle_{z}\right)\left(\left\langle\bar{u}_{t}\right\rangle_{z}-U_{2}\right) d y
$$

where $\bar{u}_{t}$ is the mean streamwise velocity. In order to avoid artificial confinement of the flow, the spanwise domain extent must be ten times the maximum momentum thickness at the far downstream end of the computational domain [47]. In the reference experiment, it was shown that at $x / \theta_{i}=1630$, the momentum thickness was $\theta / \theta_{i} \approx 39$, and the simulation spanwise domain extent of $L_{z}=392 \theta_{i}$ prevents flow confinement. The cross-stream domain size matches that of the experimental facility.

The mesh sensitivity of the mixing layer has been studied extensively elsewhere [30]. As this previous study has shown that a well-designed LES mesh can produce accurate mixing layer simulations, the mesh used in that study forms the basis of the grid employed here. The domain is discretised into $768 \times 256 \times 256$ cells. The streamwise grid spacing at the inflow plane is $\Delta x / \theta_{i}=0.46$. The cross-stream grid spacing in the plane of the splitter plate is $\Delta y / \theta_{i}=0.086$. Grid-stretching is employed in the streamwise and cross-stream directions in order to reduce the overall cell count and maintain a reasonable computational cost for the simulation. The upper boundary 
layer is resolved with 36 cells, and the lower boundary layer is resolved with 48 cells. The spanwise grid points are evenly distributed in this direction.

The imposed inflow boundary condition used in Case LWN corresponds to that commonly used in the simulation of the spatially-developing mixing layer originating from laminar initial conditions [30], [34]. The inflow velocity profile, and associated velocity fluctuations are shown in Figure 1 for the high-speed stream, with similar profiles used to prescribe the low-speed stream.

\subsection{Case $L C F$}

The main mixing layer domain extent and resolution in Case LCF matches that of Case LWN described above. However, to produce the inflow condition for the mixing layer simulation, so-called 'virtual blocks' are placed upstream of the splitter plate trailing edge in both streams. The virtual blocks encompass a region $14 \delta$ upstream of the trailing edge of the splitter plate, where $\delta$ is the high-speed side boundary-layer thickness at the splitter plate trailing edge. In both streams, the recycling plane is located $1 \delta$ upstream of the end of the virtual domain. In each stream, the vertical domain extends from the splitter plate to the guidewall of the domain, and the splitter plate is modelled through a no-slip boundary condition. The outer guidewall in each virtual block is modelled with a slip boundary condition. The mesh resolution in the virtual domains matches that of initial region of the mixing layer domain. 


\section{Results}

The fixed lateral walls of both the experimental rig and the computational domain results in the presence of an adverse pressure gradient in the flow. This pressure gradient results in a reduction of the low-speed freestream velocity with increasing streamwise distance from the splitter plate. For consistency with the experimental data, the local freestream velocity values are used when velocity data are normalised by $\Delta U=\left(U_{1}-U_{2}\right)$.

\subsection{Flow Statistics}

The momentum thickness variation of each simulation is shown in Figure 2. The tailing-off of the momentum thickness at the downstream end of the computational domain is caused by the passage of continuously growing structures through the outflow plane. As the structure passes through the outflow plane, the entrainment pattern into the structure changes, resulting in a reduction in the growth of the mixing layer. This type of tailing-off is common in other simulations of plane mixing layers [26], [30] and jet shear layers [46]. Although both cases originate from matching inflow profiles, the simulations do not produce matching profiles for the momentum thickness distribution. In the initial region, Case LCF grows slightly more quickly than Case LWN, whilst in the self-similar region, the growth rate of Case LCF is higher than that of Case LWN. It should be noted, however, that both simulations show generally good agreement with the experimental data.

The momentum thickness growth rate can be determined from the linear gradient in the momentum thickness distribution. For Case LWN, this takes

a value of $\mathrm{d} \theta / \mathrm{d} x=0.0209$, and for Case LCF $\mathrm{d} \theta / \mathrm{d} x=0.0238$. When 
compared to experimental data, Case LWN under-predicts the growth rate by approximately 14\%, whilst Case LCF produces almost matching data.

An alternative measure of the growth of the mixing layer can be obtained from the visual thickness. Sequences of instantaneous spanwise-averaged passive scalar flow visualisation are overlaid, and the wedge shape traced out by the $\langle\bar{\xi}\rangle_{z}=0.01,0.99$ scalar lines is determined. This so-called $1 \%$ thickness of the flow is then related to the visual thickness growth rate by

$$
\delta_{v i z}^{\prime}=\frac{\delta_{v i z}}{x-x_{0}}=k R
$$

where $k$ is a constant, and $x_{0}$ is the virtual origin of the flow. For Case LWN, the growth rate constant is determined as $k \approx 0.32$ - this value has been reported in other mixing layers with idealised white-noise fluctuation environments and is linked to the continuous linear growth of coherent structures [30]. For Case LCF the growth rate constant is $k \approx 0.366$, almost $15 \%$ higher than Case LWN. The visual thickness growth rate in Case LCF is in much better agreement with the experimental data, where $k \approx 0.36$. The visual thickness growth rates of both simulations do, however, fall within the range of previous experimental data [6].

Profiles of normalised mean streamwise velocity $\left\langle\bar{u}_{t}\right\rangle_{z}$, recorded at $x / \theta_{i}=$ 1000 in the simulations, are shown in Figure 3a. Both simulations produce data which compare extremely well with the reference data. In Figure 3b, the profiles of streamwise r.m.s. velocity fluctuations, $\left\langle u_{\text {r.m.s. }}\right\rangle_{z}$, show some differences between the two simulations; Case LCF slightly over-predicts the level of fluctuation at the outer edges of the mixing layer, but otherwise both simulations produce good comparisons with the experiment. The evolution of 
the peak streamwise velocity fluctuation with streamwise distance is shown in Figure 4. Both simulations predict a peak in the fluctuation, similar to that observed experimentally. In Case LWN the peak magnitude is over-predicted, and is shifted downstream when compared with the reference data - a feature that is common in simulations originating from initially laminar conditions with imposed white noise fluctuations [27], [28], [30]. Case LCF predicts the location of the overshoot very well, but also over-predicts the peak level of the fluctuation. To within experimental uncertainty, both simulations attain statistical self-similarity in the region of $x / \theta_{i} \approx 700$, well within the $1000 \theta_{i}$ criterion suggested by Bradshaw [7].

Power Spectral Density plots of the streamwise velocity fluctuation along the mixing layer centreline at certain streamwise locations are presented in Figure 5. Near the splitter plate trailing edge at $x / \theta_{i}=4$, there is a large difference in the spectral energy in the two simulations. The extremely low energy level of the spectral plot in Case LWN shows that the pseudo-random white noise is almost completely dissipated immediately downstream of the inflow plane. This is unsurprising, as the fluctuations in this simulation are neither physically- nor temporally-correlated. At $x / \theta_{i}=45$, peaks in the spectra in both simulations can be observed. Theoretical analysis has shown that the frequency of the most amplified disturbance in a two-stream mixing layer is given by

$$
\mathrm{St} \approx \frac{f \delta_{\omega}}{4\left(U_{1}-U_{2}\right)} \approx \frac{0.017}{R}
$$

where $f$ is the frequency, and $\delta_{\omega}$ is the vorticity thickness [48]. For the current flow conditions, the most dominant frequency is estimated as $f \approx 1140 \mathrm{~Hz}$. 
In Case LWN, the peak occurs at $f=830 \mathrm{~Hz}$, whilst in Case LCF it occurs at $f=1080 \mathrm{~Hz}$. The underprediction of the fundamental instability frequency has been recorded in other simulations where white-noise fluctuation environments have been used [27], [30]. Further downstream, the roll-off in the spectra in both simulations approaches the $-5 / 3$ value indicative of a transition to turbulence. For the current flow conditions, it is expected that the flow will undergo transition to turbulence in the computational domain. Analysis of the spectral plots and side-view flow visualisations shows that the transition is precipitated by a pairing interaction between primary $\mathrm{K}-\mathrm{H}$ rollers once the Reynolds number, based on the local mixing layer visual thickness and velocity difference across it, exceeds $10^{4}$. This is in agreement with past experimental [15] and computational data [28], [30].

\subsection{Flow Structure}

The discrepancies in the flow statistics obtained from Cases LCF and LWN mean that, for a given set of inflow conditions, the simulated mixing layer is affected by the nature of the imposed disturbances. A qualitative understanding of any changes in the flow field can be obtained from visual inspection of the computed flow fields. The passive scalar, $\bar{\xi}$, is used to produce flow visualisation images that are analogous to experimental schlieren photographs. As the current simulations are of uniform density, it is not possible to obtain a density-gradient representation of numerical schlieren. Instead, visualisation of the spanwise-averaged passive scalar, $\langle\bar{\xi}\rangle_{z}$, is used to reveal the structure in the mixing layer flow. It has been shown elsewhere that spanwise-averaged scalar images reveal the presence of coherent structures in the turbulent flow [28], [30], [33]. 
Typical instantaneous, spanwise-averaged scalar distributions are shown in Figures 6 and 7 for Cases LWN and LCF respectively. It is clear that both simulated mixing layers contain large-scale vortex structure. In the initial region downstream of the splitter plate trailing edge, both mixing layers contain laminar vortices which form owing to the primary instability of the flow. Further downstream the mixing transition occurs as a result of a pairing interaction between primary vortices, and turbulent coherent structures occupy the full visual thickness of the mixing layer. In the pretransition region, the spanwise averaged scalar distribution in the vortices is similar in both cases. The spanwise-averaged scalar distribution in the posttransition structures, however, does show qualitative differences between the two simulations. In Case LWN, the scalar lines in the interconnecting braid regions are quite widely spaced, with the structure cores made visible by the 'bumps' in the scalar lines on the upper and lower sides of the flow between the braids. In Case LCF, however, the structure cores are much more readily visible, and the scalar lines in the braid regions are much more tightly packed together. This disparity in the scalar distribution in the braids has been observed in temporal mixing layer simulations, where two-dimensional disturbances imposed on the initial condition resulted in a regular array of spanwise coherent structures and well-organised streamwise rib vortices [49].

Spanwise averaging of the scalar field produces an integrated representation of a three-dimensional flow-field. It is not possible, therefore, to draw any conclusions on the underlying three-dimensional flow structure from these images. Qualitative evidence of the presence of streamwise vorticity in the flow can be obtained from analysis of cross-stream $y-z$ planes of the passive 
scalar distribution. This analysis of simulation data is analogous to visual inspection Planar Laser Induced Fluorescence (PLIF) images, which has been used to infer the nature of streamwise vorticity in experiments [17], [50]. A typical pre-transition K-H vortex in each simulation is shown in Figure 8. The spanwise-averaged scalar distribution in both vortices has the typical 'swiss-roll' pattern, with tongues of unmixed fluid penetrating into the flow around a centre of rotation. The centre of rotation of each vortex in Figure 8 is in the plane of $x / \theta_{i}=108$. The $y-z$ distribution through this plane is shown in Figure 9 for each vortex in Figure 8. There is a dramatic difference in the scalar distribution across the span of the primary vortices in the two simulations. In Case LWN tongues of pure un-mixed fluid extend far across the vertical extent of the layer. The pattern of the passive scalar however, bears little qualitative resemblance to the 'mushroom-shaped eruptions' that characterise the presence of streamwise vorticity observed in experiments [17]. Analysis of sequences of flow visualisation images in Case LWN demonstrates that the secondary instability forms through the kinking of a primary roller, which then connects with its downstream neighbour to form a set of streamwise vortices. The spanwise location at which the formation of streamwise vortices occur varies from cycle to cycle. This type of 'localised pairing' has commonly been observed in simulations where the background disturbance environment has been provided by random fluctuations [24], [51]. From the $y-z$ distribution in Case LCF, in contrast, it can be inferred from the scalar distribution that two rows of counter rotating vortices are already present in the flow. Sequences of visualisation output at this location show that the streamwise vortex structure is wrapped around the primary $\mathrm{K}-\mathrm{H}$ roller in a 
configuration which matches the schematic proposed by Bernal and Roshko $[17]$.

Representative post-transition coherent structures in both simulations are shown in Figure 10. In the spanwise-averaged view, the structures have a qualitatively similar distribution with a well-defined structure core surrounded by an upstream and downstream interconnecting braid. The $y-z$ cuts through the centre of rotation of the structures, located at $x / \theta_{i}=650$, (Figure 11) also share similar features. Two rows of 'mushroom-shaped' eruptions are present on the upper and lower sides of the coherent structure. The secondary structure has therefore developed in Case LWN, and it is also evident that in both simulations the streamwise vortices survive beyond the mixing transition and into the fully turbulent flow beyond. Qualitatively the image is very similar to the high Reynolds number PLIF visualisation of Jimenez et al. [50].

\subsection{Streamwise Vortices}

The images presented in Figures 9 and 11 provide qualitative evidence for the presence of a streamwise vortex structure in the mixing layer. A more quantitative measure of the streamwise structure can be obtained from measurements of mean flow quantities across the span of the mixing layer. Up to 960 cross-stream planes are recorded at several streamwise measurement stations. The locations of these measurement stations are given in Table 2 , and are evaluated in terms of the raw distance from the splitter plate trailing edge, the normalised streamwise distance $x / \theta_{i}$, and the pairing parameter, $x_{i}^{*}=R x /\left(30 \theta_{i}\right)$. From these samples, mean statistical information is obtained and analysed for the presence of streamwise vortices. 


\begin{tabular}{ccc}
$x(\mathrm{~m})$ & $x / \theta_{i}$ & $x_{i}^{*}$ \\
\hline 0.02 & 44 & 0.96 \\
0.05 & 108 & 2.4 \\
0.1 & 217 & 4.8 \\
0.15 & 326 & 7.2 \\
0.3 & 652 & 14.4 \\
0.45 & 978 & 21.5 \\
0.6 & 1304 & 28.8 \\
\hline
\end{tabular}

Table 2: Cross-stream measurement locations.

The spanwise variation of the mean streamwise velocity along the plane of the splitter plate $\left(y / \theta_{i}=0\right)$ at each measurement station is shown in Figure 12. In Case LWN, the distribution of the mean velocity is fairly flat, with some low-level fluctuations visible in the profiles. The maximum peakto-peak amplitude of the variation is approximately $6 \%$, substantially lower than the variations obtained experimentally for plane mixing layers [14], [16] [18]. Furthermore, there is no obvious evidence for a regular wavelength in the variation of the profiles at any downstream location. This implies that, in a time-averaged sense, the streamwise vorticity in Case LWN is not statistically stationary.

Case LCF, in contrast, shows significant regular variations in the mean velocity across the span, with the spanwise wavelength of the oscillation increasing with downstream distance. The maximum peak-to-peak amplitude in this case is approximately $20 \%$, similar to that reported in the experiments of Huang \& Ho [14], who conducted experiments in the same experimental 
facility as Browand and Latigo [4]. This value is also in the range of that reported by Jimenez (40\%) [18], and by Bell and Metha (10\%) [16].

The spanwise wrinkling of the mean velocity field in Case LCF shows very similar behaviour to that obtained in the experiments of Bell \& Mehta [16] and Huang \& Ho [14], who independently examined streamwise vortex structure in mixing layers of comparable flow conditions. The wrinkling of the mean streamwise velocity corresponds to the presence of stationary streamwise vorticity in the flow. Inspection of Figure 12 shows that the number of peaks and troughs in Case LCF decreases with increasing downstream distance. It can be concluded that the number density of the streamwise vortices decreases as the flow evolves downstream, in qualitative agreement with the data of Bell \& Mehta [16].

Experimental evidence indicates that the thickness of the mixing layer can be substantially affected by the wrinkling of the flow, in the presence of stationary streamwise vortices [16]. The thickness of the mixing layer is computed from the vertical distance between the locations where $U_{0.01}=$ $U_{2}+0.01\left(U_{1}-U_{2}\right)$, and $U_{0.99}=U_{2}+0.99\left(U_{1}-U_{2}\right)$. The standard deviation of the mixing layer thickness across the span of the mixing layer is shown in Figure 13, along with comparable experimental data of Bell \& Mehta [16]. The data are presented in terms of the pairing parameter in order to provide a consistent non-dimensional streamwise scale between the simulation and experimental data. The agreement between Case LCF and the experimental data is remarkable. The large peak in the variation of mixing layer thickness at $x_{i}^{*} \approx 2.4$ implies that the streamwise vortices are rapidly amplified in this region of the flow. A value of $x_{i}^{*}=2$ is the location, on average, of 
the roll-up of the mixing layer into $\mathrm{K}-\mathrm{H}$ vortices, and it appears that the amplification of the streamwise vorticity occurs following the emergence of the primary vortex structure. Further downstream the variation in visual thickness reaches a near constant level of $\sim 4 \%$, suggesting that the flow has become more-or-less 'two-dimensional'. In Case LWN by contrast, the peak variation in mixing layer thickness occurs further downstream at a pairing parameter value of $x_{i}^{*} \approx 5$, in the region where vortex pairing events take place. The magnitude of the peak is significantly lower than both case LCF and the experimental data. Further downstream the variation maintains a magnitude of $\sim 3 \%$, suggesting that the flow is more-or-less statistically two-dimensional.

Secondary shear stress contour maps, $\overline{u^{\prime} w^{\prime}}$, normalised by $\Delta U^{2}$, are shown at five of the streamwise measurement stations in Figures 14 \& 15 for Cases LWN and LCF respectively. Direct experimental measurement of this quantity has shown that alternating bands of the secondary shear stress along the spanwise direction indicates the presence of statistically stationary streamwise vorticity [16]. In Case LWN, the levels of secondary shear stress at the first measurement station (Figure 14a) are negligible. The white noise inflow disturbances do not generate organised streamwise vorticity upstream of the emergence of primary K-H rollers. At the second and third measurement stations (Figures 14b \& 14c), some banding of the $\overline{u^{\prime} w^{\prime}}$ contours is evident, but it is irregular in its structure. Further downstream at $x / \theta_{i}=652,1304$ (Figures $14 \mathrm{~d} \& 14 \mathrm{e}$ ), there is again no obvious banding of the secondary shear stress. These ensemble averaged maps, coupled with the statistics presented in Figure 12 \& 13 infer that statistically stationary streamwise vorticity does 
not form in a simulation where the disturbance environment is provided by pseudo-random white noise.

In Case LCF, the secondary shear stress contour map at the first measurement station (Figure 15a) reveals the presence of irregular clusters of this quantity located across the span of the mixing layer. At a given spanwise location, $\overline{u^{\prime} w^{\prime}}$ alternates on the high- and low- speed stream, with the sign of $\overline{u^{\prime} w^{\prime}}$ in each stream varying across the span. The magnitude of this organised secondary shear stress at this measurement station in Case LCF is two orders of magnitude higher than that found in Case LWN. At the second measurement station (Figure 15b), an alternating pattern in the secondary shear stress is visible. At a given spanwise location, a cluster of $\overline{u^{\prime} w^{\prime}}$ is flanked by neighbouring clusters of opposite sign. These distributions in the near-field region have also been observed experimentally [16]. There is some correlation between the locations of the clusters at $x / \theta_{i}=44$, and the bands of secondary shear stress at $x / \theta_{i}=108$; it appears that the residual streamwise vorticity in the initial region of the flow is amplified into a single row of structures. With reference to Table 2, these two measurement stations are located at $x_{i}^{*}=0.96,2.4$ respectively. The flow rolls up into primary K-H rollers at $x_{i}^{*} \approx 2$, and it therefore can be deduced that the amplification of the residual streamwise vorticity in the separating boundary layers is amplified into a single row of streamwise vortices by this roll-up process.

At $x / \theta_{i}=217$ (Figure 15c), a single row of alternating bands of secondary shear stress has developed. The bands present at this measurement station can be traced upstream to the previous measurement station. Further downstream at $x / \theta_{i}=652$ in Figure $15 \mathrm{~d}$ the scale of the alternating bands of $\overline{u^{\prime} w^{\prime}}$ 
has increased, but it is still possible to trace back some of the bands to the upstream measuring stations, particularly at $z / \theta_{i}=320,350$ and 380. Some form of interaction has taken place in the region of $z / \theta_{i}=240$, such that this region is now occupied by a single, large streamwise vortex. At the far downstream measuring station (Figure 15e) bands of alternating sign $\overline{u^{\prime} w^{\prime}}$ are still visible. The number density of these bands of secondary shear stress has decreased, and their spacing has increased significantly. Again, the vortices at $z / \theta_{i}=170,240$, and 310 in Figure 15e can easily be traced back to corresponding structures in Figure 15d. The evidence in this simulation indicates that these statistically stationary streamwise vortices persist far into what is normally considered the fully-developed flow, and that their size and spacing increases with streamwise distance. These findings are in agreement with experimental evidence [16].

\section{Discussion}

The results presented in Section 4 demonstrate that the simulated plane turbulent mixing layer exhibits a hypersensitivity to the nature of the imposed inflow condition. It should be noted that no effort has been made to assess the effect of the magnitude of the initial fluctuation level on the mixing layer evolution, as this would represent a much larger study that is beyond the focus of this article.

A typical description of the mixing layer initial conditions consists of the freestream velocities, the boundary layer profiles and associated integral properties, and a measure of the background freestream turbulence fluctua-

tion level. Both cases LCF and LWN develop from what are, statistically at 
least, matching initial conditions. The marked differences in the evolution of the simulations and the nature of the streamwise vorticity contained within them warrant further discussion.

The laboratory mixing layer develops in a wind tunnel where the background turbulence fluctuations are both spatially- and temporally-correlated. Case LCF produces a simulation where the fluctuations at the trailing edge of the splitter plate are also physically-correlated. From the secondary shear stress contour maps it can be inferred that low-level streamwise vorticity is present in the mixing layer immediately downstream of the splitter plate trailing edge in Case LCF, which is then rapidly amplified as the flow proceeds downstream. The secondary shear stress contours of Figures 15 show that these streamwise vortices are statistically stationary, and their number density decreases with increasing distance from the splitter plate. The presence of these streamwise vortices in the mixing layer has a quantifiable effect on the mixing layer growth rate, and on the evolution of the turbulence statistics of the flow. The persistence of the stationary streamwise vortices far into the self-similar region is in agreement with the experimental observations of Bell \& Mehta [16] for comparable flow conditions, and provide further evidence that the criterion for self-similarity may need to be re-assessed.

The geometrical simplicity of a plane mixing layer means that the flow configuration is attractive for numerical simulation. Current supercomputing power has rendered DNS of the spatially-developing mixing layer computationally feasible. Direct Numerical Simulation does, of course, permit the study of flows without particular inflow condition features that could be considered unique to experiments, permitting the use of simplified boundary 
conditions in initially-laminar mixing layer. To date, all DNS studies of the spatially-developing mixing layer have originated from such simplified inflow boundary conditions, of both hyperbolic tangent [26], [27] or Blasius profile form [34]. In these simulations, the imposed fluctuations are obtained from random fluctuations, similar in form to that of Case LWN. The flow visualisation images presented from these studies show no evidence of organised streamwise vorticity in the region immediately downstream of the splitter plate. Rather, it appears as though the streamwise vortex structure is generated through localised pairings of the primary K-H rollers. DNS studies are, by definition, free from turbulence modelling assumptions as all scales of motion are resolved in the simulation. This does not mean, however, that the DNS mixing layer is insensitive to the imposed inflow condition. The results presented here demonstrate that imposing physically-correlated, lowlevel fluctuations in the separating boundary layers results in the formation of statistically stationary streamwise vortices, which in turn influences the obtained mixing layer flow statistics. As DNS is increasingly used to gain insights into flow dynamics, it must be of paramount importance to impose physically realistic inflow conditions (including fluctuations) to ensure that the simulated mixing layer is an accurate representation of a real laboratory flow.

As noted above, a classical documentation of the initial condition of the mixing layer usually provides a freestream turbulence fluctuation level, expressed as a percentage of the freestream velocity. There are very few published experimental datasets that detail the fluctuation level in the laminar boundary layer [4], [45] and, to the authors' knowledge, no experimental 
study has documented the fluctuation profiles of all three velocity components at the splitter plate trailing edge. Given that the reference experiment [4] did not record profiles of $v_{r . m . s .}$ or $w_{r . m . s}$ in the boundary layer, these profiles have been assumed in this study. It is well known that the mixing layer displays a hypersensitivity to its initial conditions, and it is not unreasonable to suggest that changes in the boundary layer fluctuation levels will produce a change in the streamwise vortex distribution. Evidence for this hyper-sensitivity has been provided in experiments where, on changing the freestreams between legs in the laboratory wind tunnel, changes in the streamwise vortex distribution were recorded [52]. It is clear that future mixing layer experiments should include a complete description of boundary layer mean velocity and velocity fluctuation profiles, as this will provide sufficient information for accurate numerical simulation studies of the flow.

\section{Conclusions}

The spatially-developing mixing layer has been studied using Large Eddy Simulation. The effect of the nature of the fluctuations imposed on the inflow condition has been assessed. A white-noise disturbance environment imposed on the inflow condition results in a simulated flow which produces reasonable mean flow statistics, but does not contain statistically stationary secondary streamwise vorticity. A correlated fluctuation environment results in a simulated mixing layer which has improved agreement with reference experimental data, and displays evidence of stationary secondary streamwise vortices across the span of the mixing layer. The variation in the mixing layer thickness caused by the presence of the stationary streamwise vortices shows 
good agreement with comparable experimental data. As the secondary flow structure has a significant impact on the near-field evolution of the mixing layer, it is very important that future DNS mixing layer databases include correlated inflow perturbations in order to recreate this structure. Furthermore, it is essential that future experiments conducted on the mixing layer provide a complete description of the fluctuation profiles in the separating boundary layers, in order to permit accurate numerical simulation of the real mixing layer flow.

\section{Acknowledgements}

The simulations presented in that article were performed on ALICE, the University of Leicester High Performance Computing facility. SJG is supported by a Senior Research Fellowship of the Royal Academy of Engineering, funded by the Leverhulme Trust.

[1] Liepmann, H. W., and Laufer, J. 1947. "Investigation of free turbulence mixing." NACA tech. note No. 127.

[2] Wygnanski, I., and Fiedler, H. E. 1970. "The two-dimensional mixing region." Journal of Fluid Mechanics, Vol. 41, pp. 327-361.

[3] Yule, A.J., 1971. "Two-dimensional self-preserving turbulent mixing layers at different free stream velocity ratios." Aeronautical Research Council Research and Memoranda, No. 3683.

[4] Browand, F. K., and Latigo, B. O. 1979. "Growth of the two-dimensional mixing layer from a turbulent and nonturbulent boundary layer." Physics of Fluids, Vol. 22, pp. 1011-1019. 
[5] Slessor, M. D., Bond, C. L., and Dimotakis, P. E. 1998. "Turbulent shear-layer mixing at high Reynolds numbers: effects of inflow conditions" Journal of Fluid Mechanics, Vol. 375, pp. 115-138.

[6] Brown, G. L., and Roshko, A., 1974. "On density effects and large structure in turbulent mixing layers." Journal of Fluid Mechanics, Vol. 64, pp. $755-816$.

[7] Bradshaw, P., 1966. "The effect of initial conditions on the development of a free shear layer. Journal of Fluid Mechanics, Vol. 26, pp. 225-236.

[8] Winant, C. D., and Browand, F. K., 1974. "Vortex Pairing: The mechanism of turbulent mixing layer growth at moderate Reynolds numbers." Journal of Fluid. Mechanics, Vol 63, pp. 237-255.

[9] Bernal, L.P., 1981. "The coherent structure of turbulent mixing layers." PhD Thesis, California Institute of Technology.

[10] Hernan, M.A., and Jimenez, J., 1982. "Computer analysis of a highspeed film of the plane turbulent mixing layer." Journal of Fluid Mechanics, Vol. 119, pp. 323-345.

[11] Dimotakis, P.E., 1986. "Two-dimensional shear layer entrainment." AIAA Journal, Vol. 24, pp. 1791-1796.

[12] D'Ovidio, A., and Coats, C. M., 2013. "Coherent-structure evolution in turbulent mixing layers. Part 1: Experimental evidence." Journal of Fluid Mechanics, Vol. 737, pp. 466-498. 
[13] Konrad, J.H., 1976. "An experimental investigation of mixing in twodimensional turbulent shear flows with applications to diffusion-limited chemical reactions." PhD Thesis, California Institute of Technology.

[14] Huang, L-S, and Ho, C-M., 1990. "Small scale transition in a plane mixing layer." Journal of Fluid Mechanics, Vol 210, pp. 475-500.

[15] Dimotakis, P. E., 2000. "The mixing transition in turbulent flows." Journal of Fluid Mechanics, Vol. 409, pp. 69-98.

[16] Bell, J. H., and Mehta, R. D., 1992. "Measurement of streamwise vortical structures in a plane mixing layer." Journal of Fluid Mechanics, Vol. 239, pp. 213-248.

[17] Bernal, L. P., and Roshko, A., 1986. "Streamwise vortex structure in plane mixing layers." Journal of Fluid Mechanics, Vol. 170, pp. 499525 .

[18] Jimenez, J., 1983. "A spanwise structure in the plane shear layer." Journal of Fluid Mechanics, Vol. 132, pp. 319-336.

[19] Lasheras, J. C., Choi, J. S., and Maxworthy, T., 1986. "On the origin and evolution of streamwise vortical structures in a plane, free shearlayer." Journal of Fluid Mechanics, Vol. 172, pp. 231-258.

[20] Pierrehumbert, R. T., and Widnall, S.E., 1982. "The two- and threedimensional instabilities of a spatially periodic shear layer." Journal of Fluid Mechanics, Vol. 114, pp. 59-82. 
[21] Moser, R. D., and Rogers, M. M., 1991. "Mixing transition and the cascade to small scales in a plane mixing layer." Physics of Fluids A, Vol. 3 pp. 1128-1134.

[22] Rogers,, M. M., and Moser, R. D., 1992. "The three-dimensional evolution of a plane mixing layer: the Kelvin-Helmholtz rollup." Journal of Fluid Mechanics, Vol. 243, pp. 183-226.

[23] Moser, R. D., and Rogers, M. M., 1993. "The three-dimensional evolution of a plane mixing layer: pairing and transition to turbulence." Journal of Fluid Mechanics, Vol. 247, pp. 275-320.

[24] Balaras, E., Piomelli, U., and Wallace, J. M., 2001. "Self-similar states in turbulent mixing layers." Journal of Fluid Mechanics, Vol. 446, pp. $1-24$.

[25] Rogers, M. M., and Moser, R. D., 1993. "Spanwise scale selection in plane mixing layers." Journal of Fluid Mechanics, Vol. 247, pp. 321337.

[26] Wang, Y., Tanahashi, M., and Miyauchi, T., 2007. "Coherent fine scale eddies in turbulence transition of spatially-developing mixing layer." International Journal of Heat and Fluid Flow, Vol. 28, pp. 1280-1290.

[27] Attili, A., and Bisetti, F., 2012. "Statistics and scaling of turbulence in a spatially developing mixing layer at $\mathrm{Re}_{\lambda}=250$." Physics of Fluids, Vol. 24, doi: 10.1063/1.2696302

[28] McMullan, W. A., Gao, S., and Coats, C. M., 2009. "The effect of inflow conditions on the transition to turbulence in Large Eddy Simulations of 
spatially developing mixing layers." International Journal of Heat and Fluid Flow, Vol. 30, pp. 1054-1066.

[29] de Bruin, I. C. C., 2001. "Direct and large-eddy simulation of the spatial turbulent mixing layer." PhD Thesis, University of Twente.

[30] McMullan, W. A., Gao, S., and Coats, C. M., 2015. "Organised large structure in the post-transition mixing layer. Part 2. Large eddy simulation." Journal of Fluid Mechanics, Vol. 762, pp. 302-343.

[31] Vreman, B., Geurts, B. and Kuerten, H., 1997. "Large eddy simulation of the turbulent mixing layer." Journal of Fluid Mechanics, Vol. 339, pp. 357-390.

[32] McMullan, W.A., 2014. "On the growth of a plane mixing layer from laminar or turbulent initial conditions." AIAA Aviation, Atlanta, GA. AIAA-2014-3096.

[33] McMullan, W. A., Gao, S., and Coats, C. M., 2007. "A comparative study of inflow conditions for two- and three-dimensional spatially developing mixing layers using Large Eddy Simulation." International Journal of Numerical Methods in Fluids, Vol. 55, pp. 589-610.

[34] Zhou, K., Attili, A., Alshaarawi, A., and Bisetti, F., 2014. "Simulation of aerosol nucleation and growth in a turbulent mixing layer." Physics of Fluids, Vol. 26, doi: 10.1063/1.4884789.

[35] Soteriou, M. C., and Ghoniem, A. F., 1998. "On the effects of the inlet boundary condition on the mixing and burning in reacting shear flows." Combustion and Flame, Vol. 112, pp. 404-417. 
[36] Soteriou, M. and Yang, X., 1999. "Inlet condition effects on particle dispersion in a shear layer." Combustion Science and Technology, Vol. 148, pp. 59-92.

[37] Xiao, F., Dianat, M., and McGuirk, J.J., 2010. "A Recycling/Rescaling Method for LES Inlet Condition Generation." Proceedings of the 8th International ERCOFTAC Symposium on Engineering Turbulence Modelling and Measurements, Marseille, France pp. 510-515.

[38] Lund, T., Wu, X., and Squires, D., 1998. "Generation of turbulent inflow data for spatially developing boundary layer simulations." Journal of Computational Physics, Vol 140, pp. 233-258.

[39] Klein, M., Sadiki, A., and Janicka, J., 2003. "A digital filter based generation of inflow data for spatially developing direct numerical or large eddy simulation." Journal of Computational Physics, Vol. 186, pp. 652665.

[40] Nicoud, F., and Ducros, F., 1999. "Subgrid-scale stress modelling based on the square of the velocity gradient tensor." Flow, Turbulence and Combustion, Vol. 62, pp. 183-200.

[41] Voke, P., and Potamitis, S. G., 1994. "Numerical simulation of a lowReynolds-number turbulent wake behind a flat plate." International Journal of Numerical Methods in Fluids, Vol. 19, pp. 377-393.

[42] Wang, P. C., and McGuirk, J. J., 2011. "Large eddy simulation of high speed nozzle flows - assessment and validation of synthetic turbulence in- 
let conditions." 20th AIAA Computational Fluid Dynamics Conference, Honolulu, HI. AIAA-2011-3555.

[43] Xiao, F., Dianat, M., and McGuirk, J. J., 2014. "LES of turbulent liquid jet primary breakup in turbulent coaxial flow." International Journal of Mulitphase Flow, Vol. 60, pp. 103-118.

[44] Hussain, A.K.M.F., and Zedan, M.F., 1978. "Effect of the initial condition on the axisymmetric free shear layer: effect of the initial fluctuation level." Physics of Fluids, Vol. 21, pp. 1475-1481.

[45] Pickett, L. M., and Ghandhi, J. B., 2002. "Passive scalar mixing in a planar shear layer with laminar and turbulent inlet conditions." Physics of Fluids, Vol. 14, pp. 985-998.

[46] Bogey, C., and Bailly, C., 2010. "Influence of nozzle-exit boundary-layer conditions on the flow and acoustic fields of initially laminar jets." Journal of Fluid Mechanics, Vol. 663, pp. 507-538.

[47] McMullan, W.A., 2015. "Spanwise Domain Effects on the Evolution of the Plane Turbulent Mixing Layer." International Journal of Computational Fluid Dynamics, Vol. 29, pp. 333-345.

[48] Monkewitz, P. A., and Huerre, P., 1982. "Influence of velocity ration on the spatial instability of mixing layers." Physics of Fluids, Vol. 25, pp. $1137-1143$.

[49] Rogers, M. M., and Moser, R. D., 1994. "Direct simulation of a selfsimilar turbulent mixing layer." Physics of Fluids, Vol. 6, pp. 903-923. 
[50] Jimenez, J., Cogollos, M., and Bernal, L. P., 1985. "A perspective view of the plane mixing layer." Journal of Fluid Mechanics, Vol. 152, pp. $125-143$.

[51] Comte, P., Sivestrini, J. H., and Bgou, P., 1998. "Streamwise vortices in large-eddy simulations of mixing layers." European Journal of Mechanics/B Fluids, Vol. 17, pp. 615-637.

[52] Plesniak, M. W., Bell, J. H., and Mehta, R. D., 1993. "Effects of small changes in initial conditions on mixing layer three-dimensionality." $E x-$ periments in Fluids, Vol. 14, pp. 286-288. 


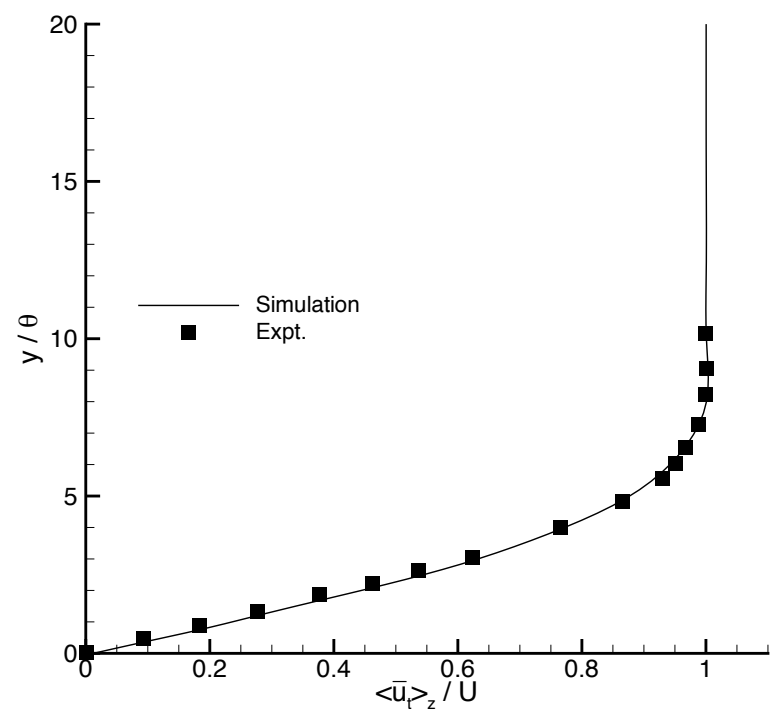

(a) Mean streamwise velocity.

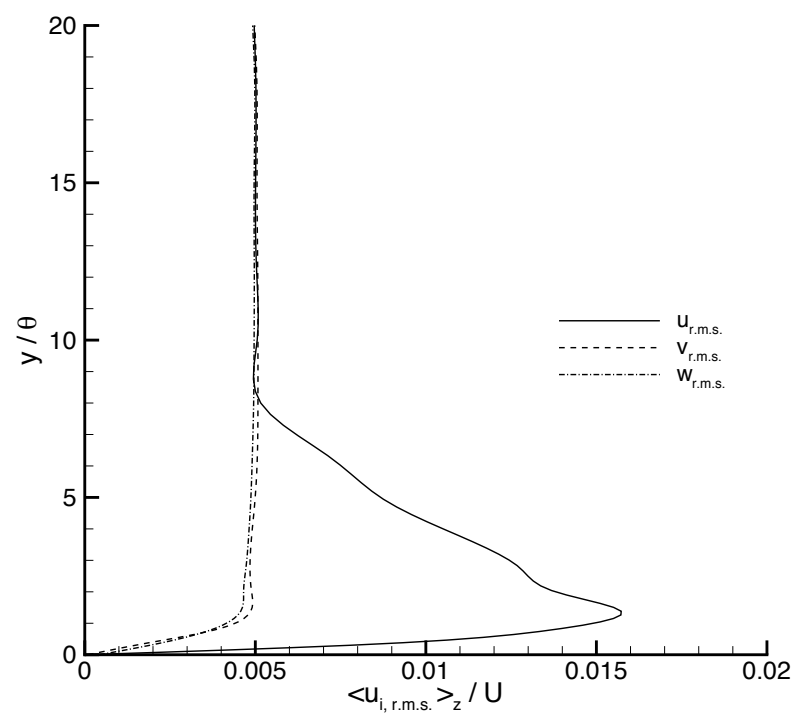

(b) Boundary layer velocity fluctuations.

Figure 1: High-speed boundary layer initial conditions in the simulations. (a) Mean streamwise velocity, (b) Streamwise velocity fluctuations 


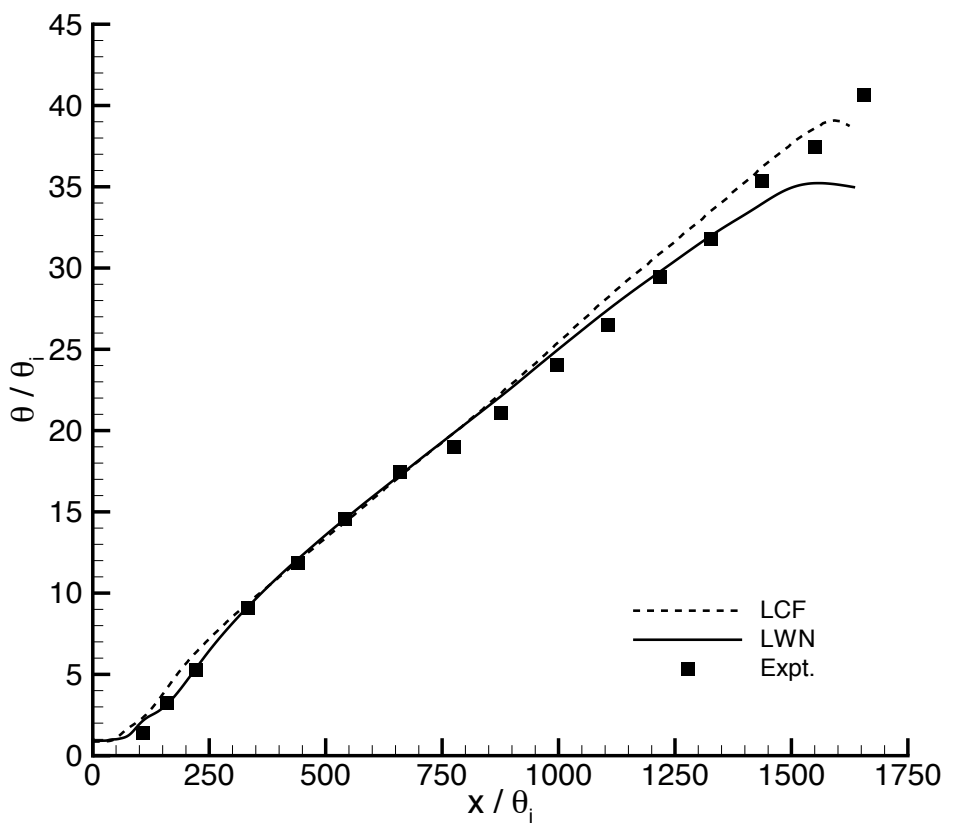

Figure 2: Normalised mixing layer momentum thickness. 


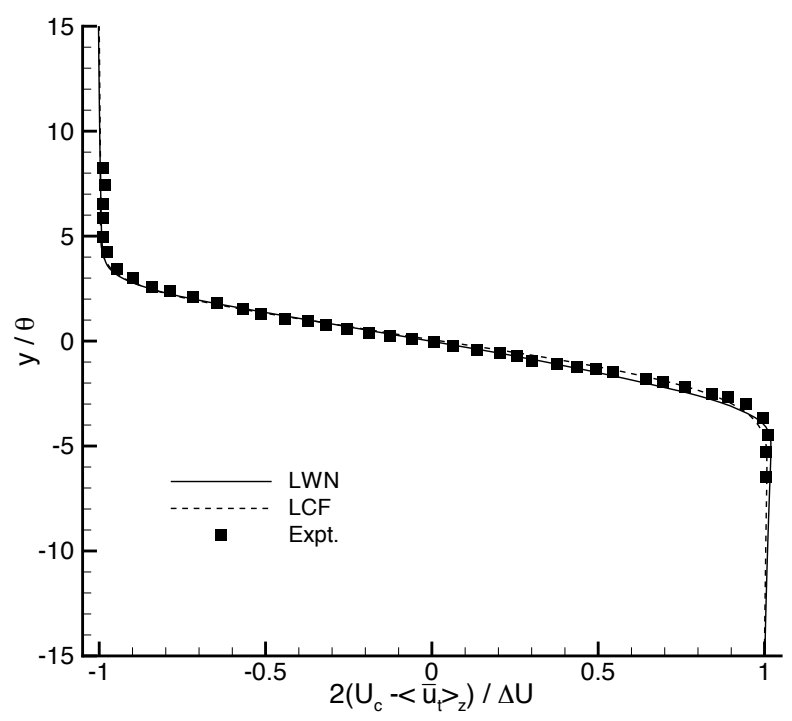

(a) Mean streamwise velocity.

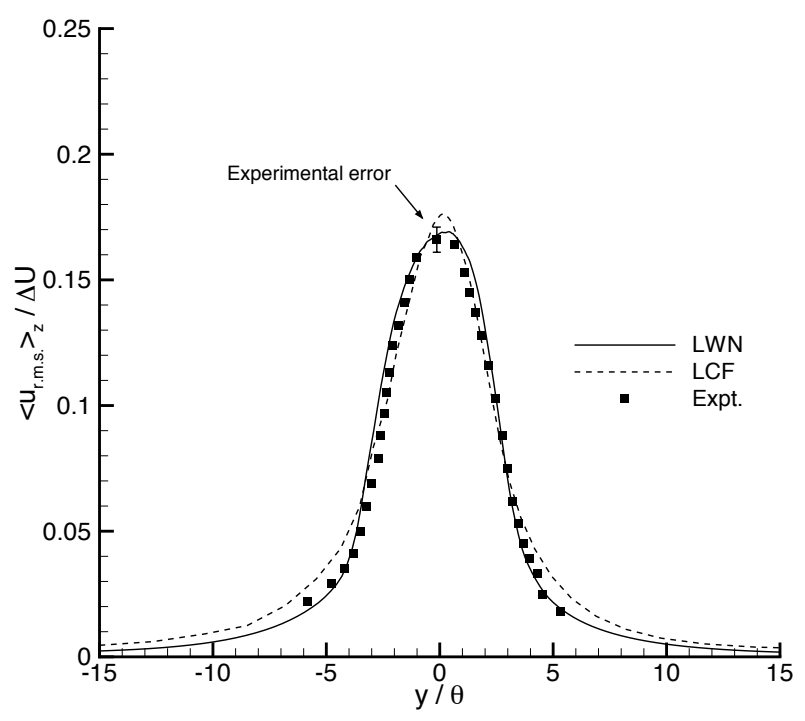

(b) Streamwise velocity fluctuations.

Figure 3: Mixing layer flow statistics recorded at $x / \theta_{i}=1000$. (a) Mean streamwise velocity, (b) Streamwise velocity fluctuations. 


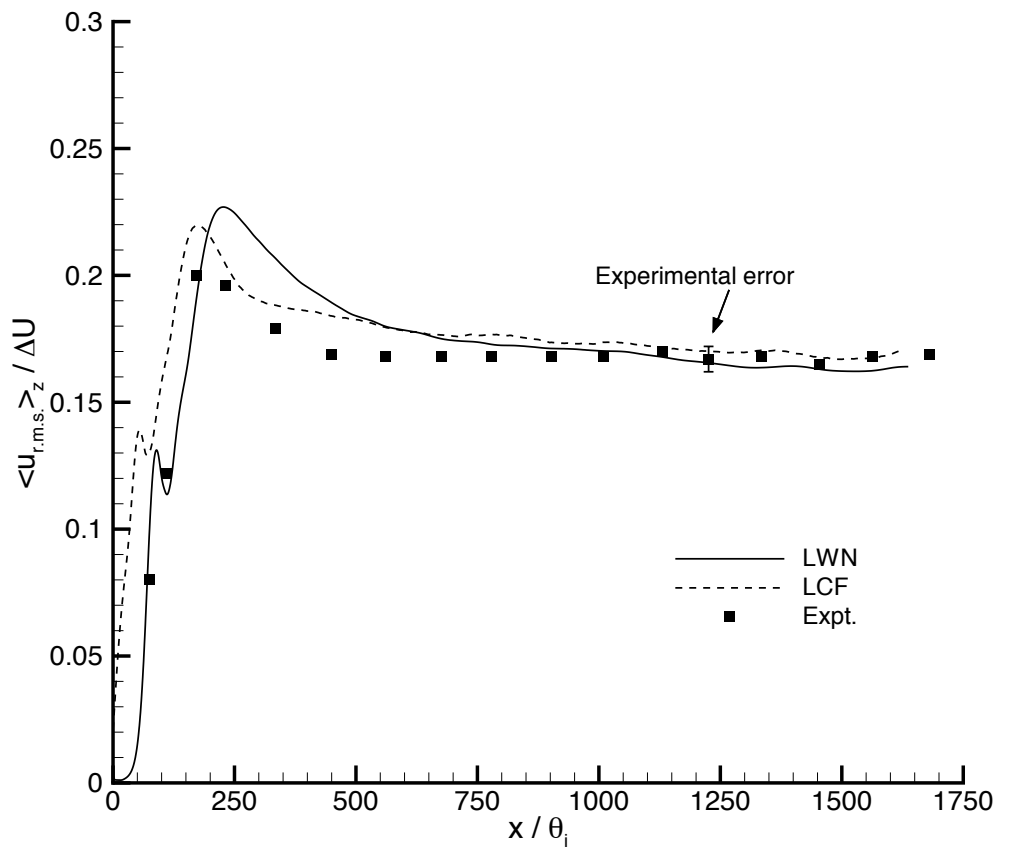

Figure 4: Streamwise evolution of maximum streamwise velocity fluctuation in the mixing layer. 


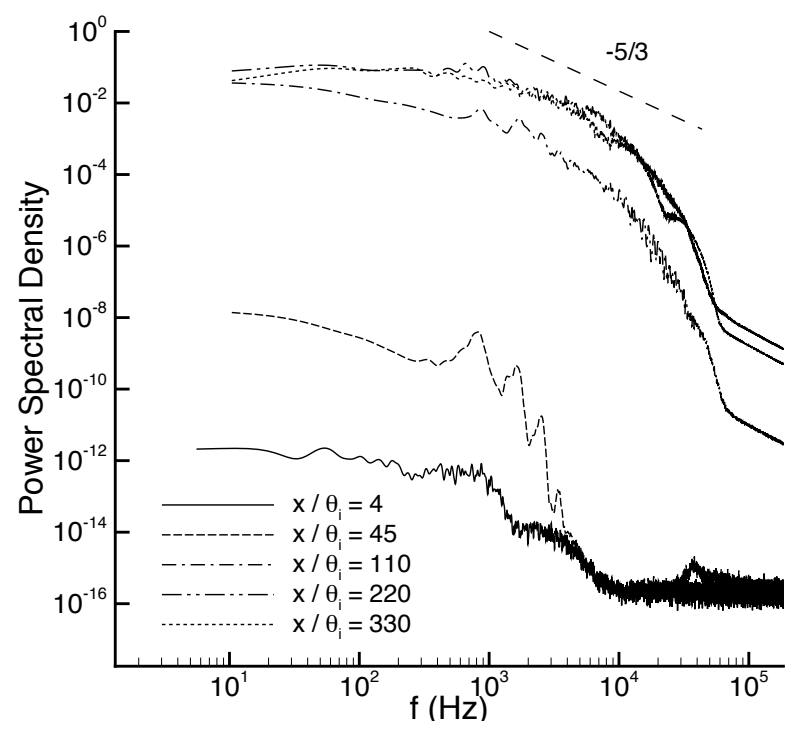

(a) LWN.

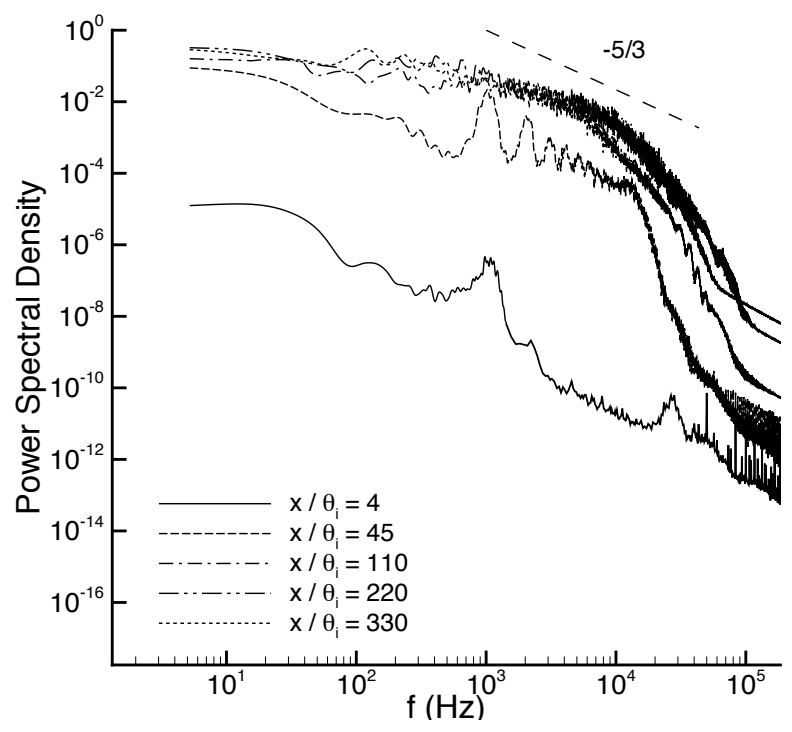

(b) LCF.

Figure 5: Streamwise velocity fluctuation power spectral density plots obtained at various streamwise locations. (a) Case LWN, (b) Case LCF 


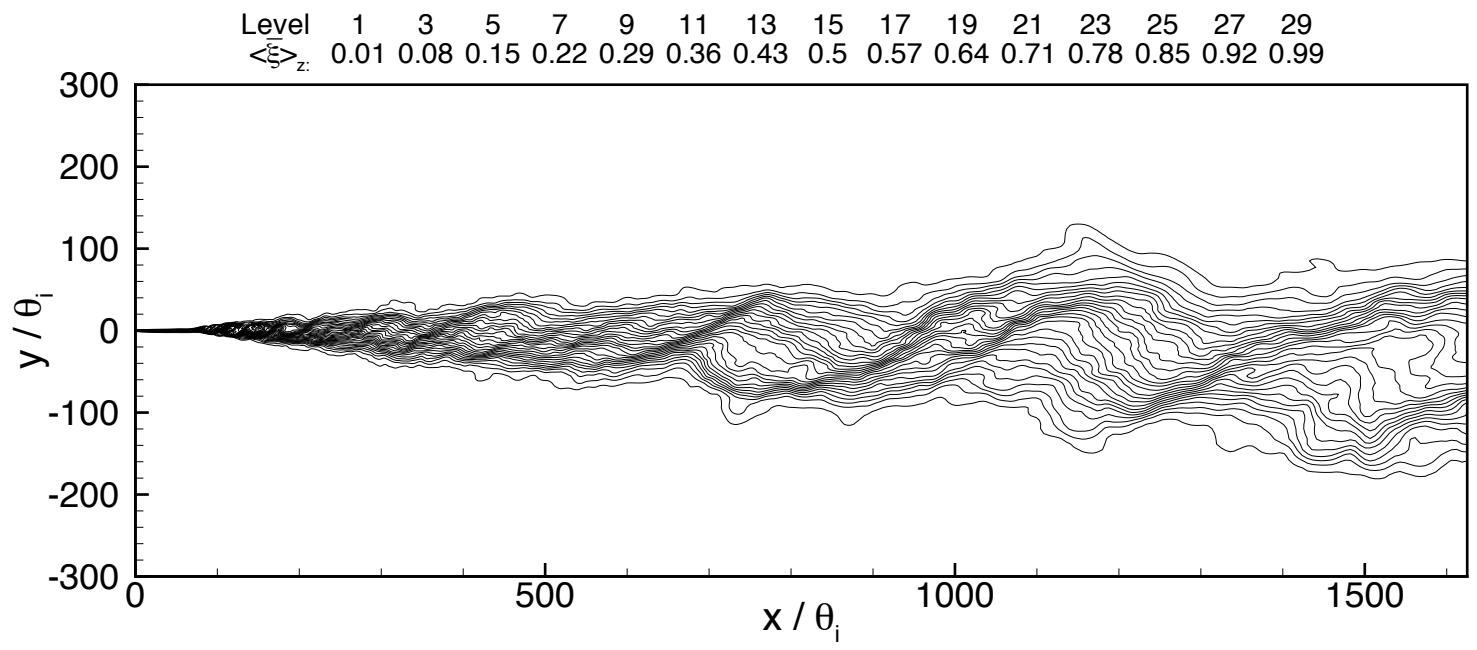

Figure 6: Instantaneous spanwise-averaged scalar distribution in Case LWN.

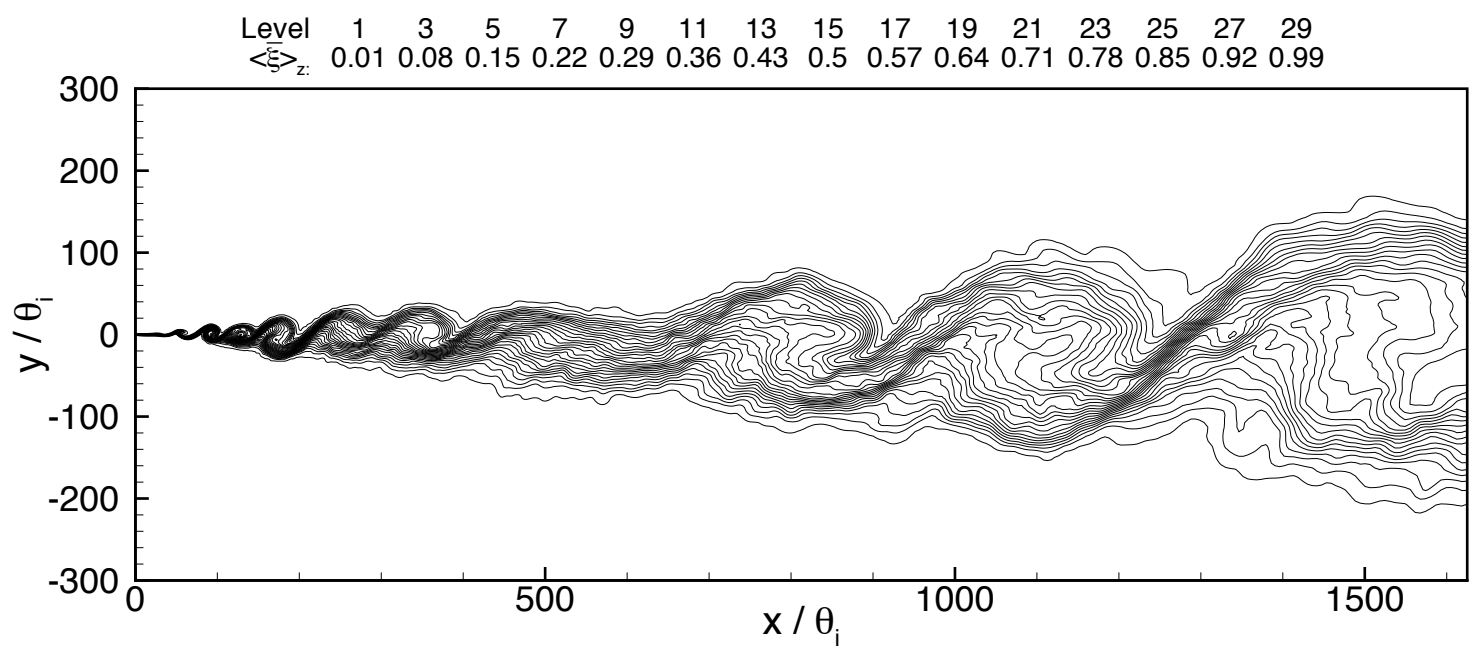

Figure 7: Instantaneous spanwise-averaged scalar distribution in Case LCF. 


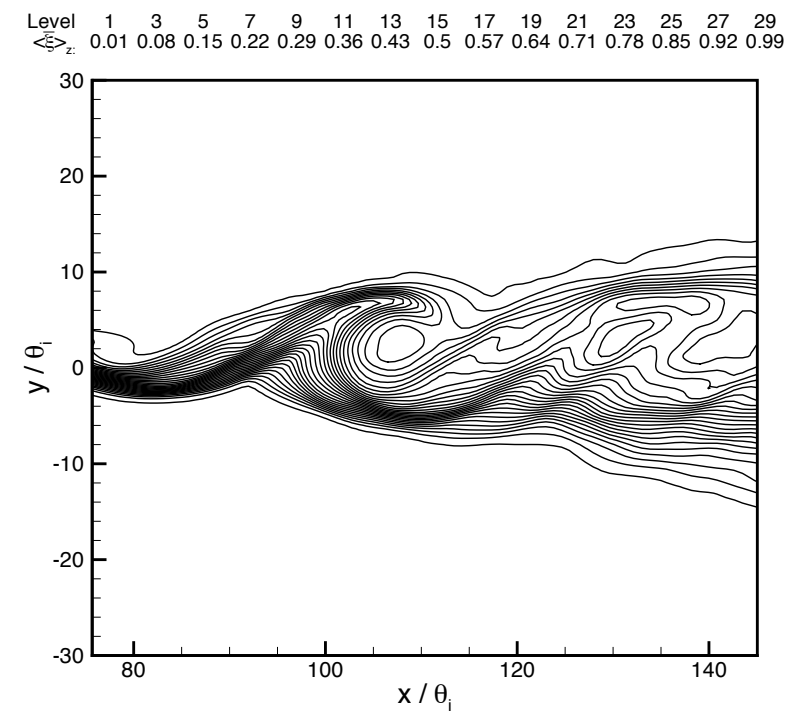

(a) Case LWN.

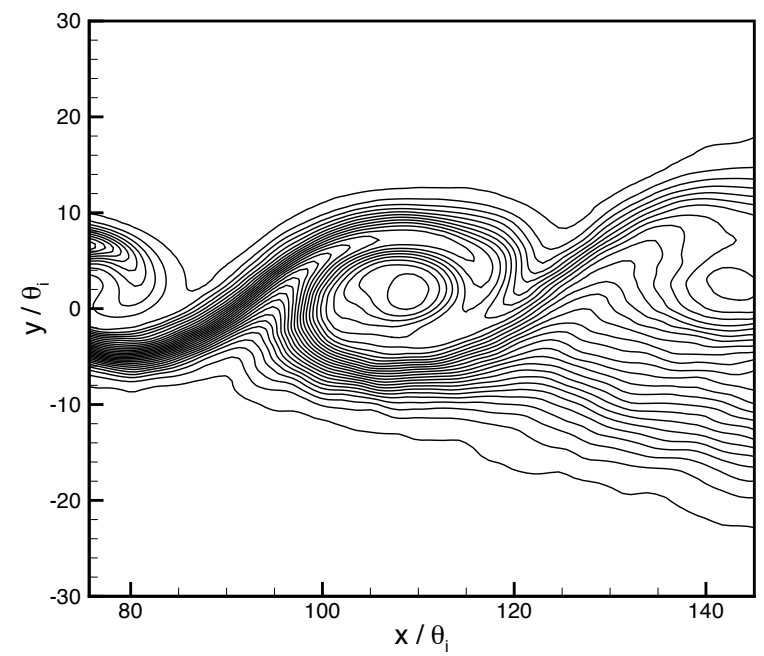

(b) Case LCF.

Figure 8: Typical pre-transition vortices. (a) Case LWN, (b) Case LCF. 


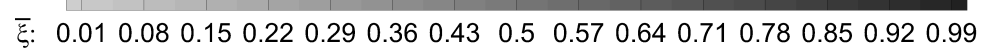

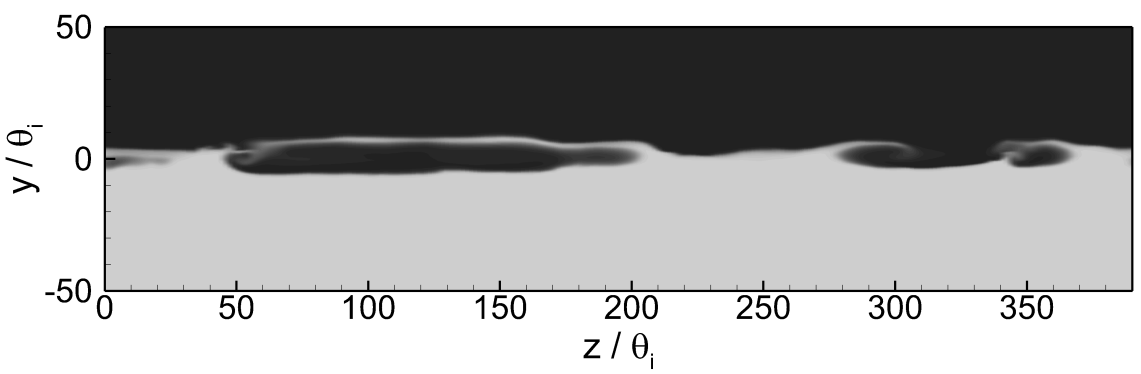

(a) Case LWN.

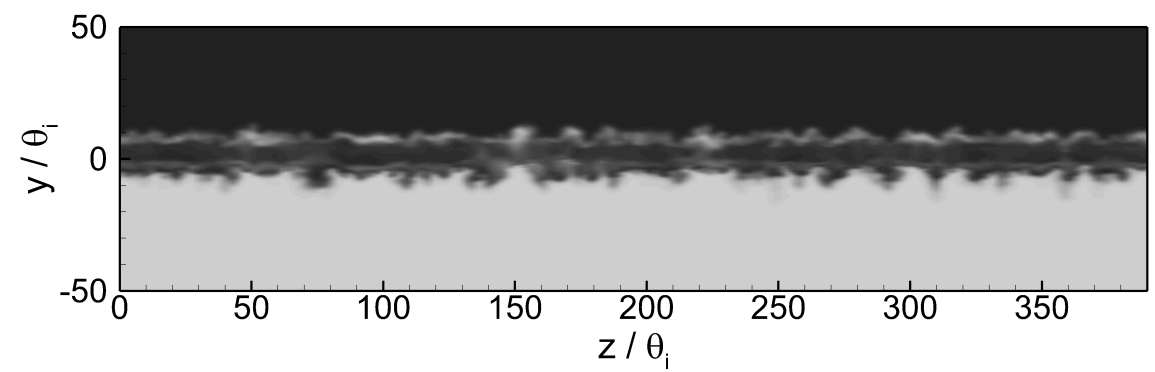

(b) Case LCF.

Figure 9: $y-z$ scalar distribution through the core of the vortices shown in Figure 8. (a) Case LWN, (b) Case LCF. 


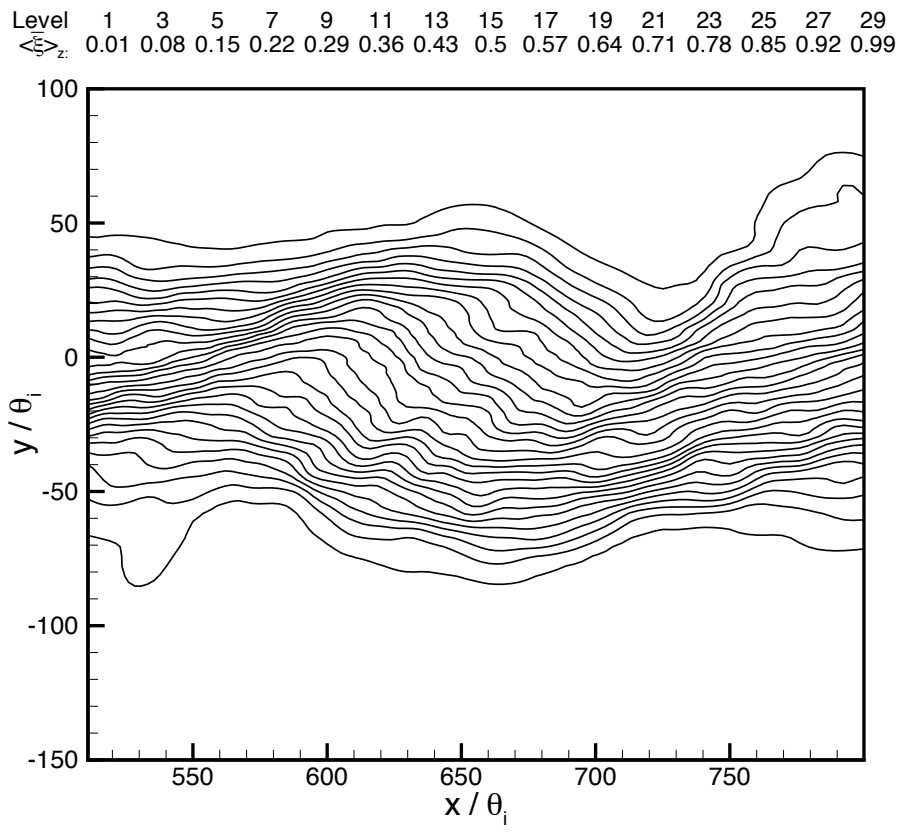

(a) Case LWN.

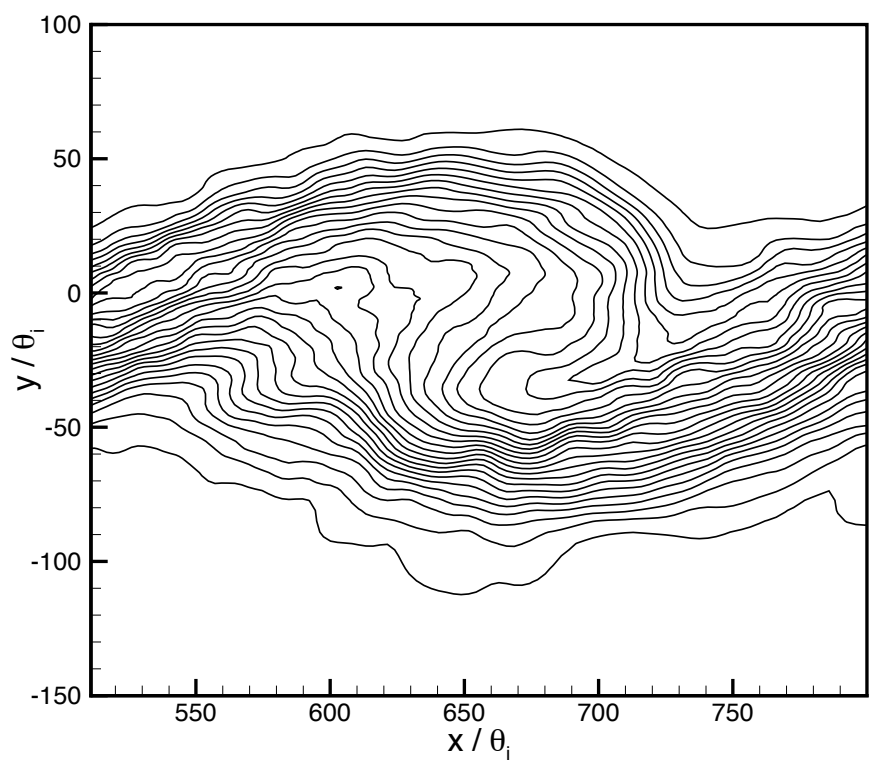

(b) Case LCF.

Figure 10: Typical post-transition coherent structures. (a) Case LWN, (b) Case LCF. 


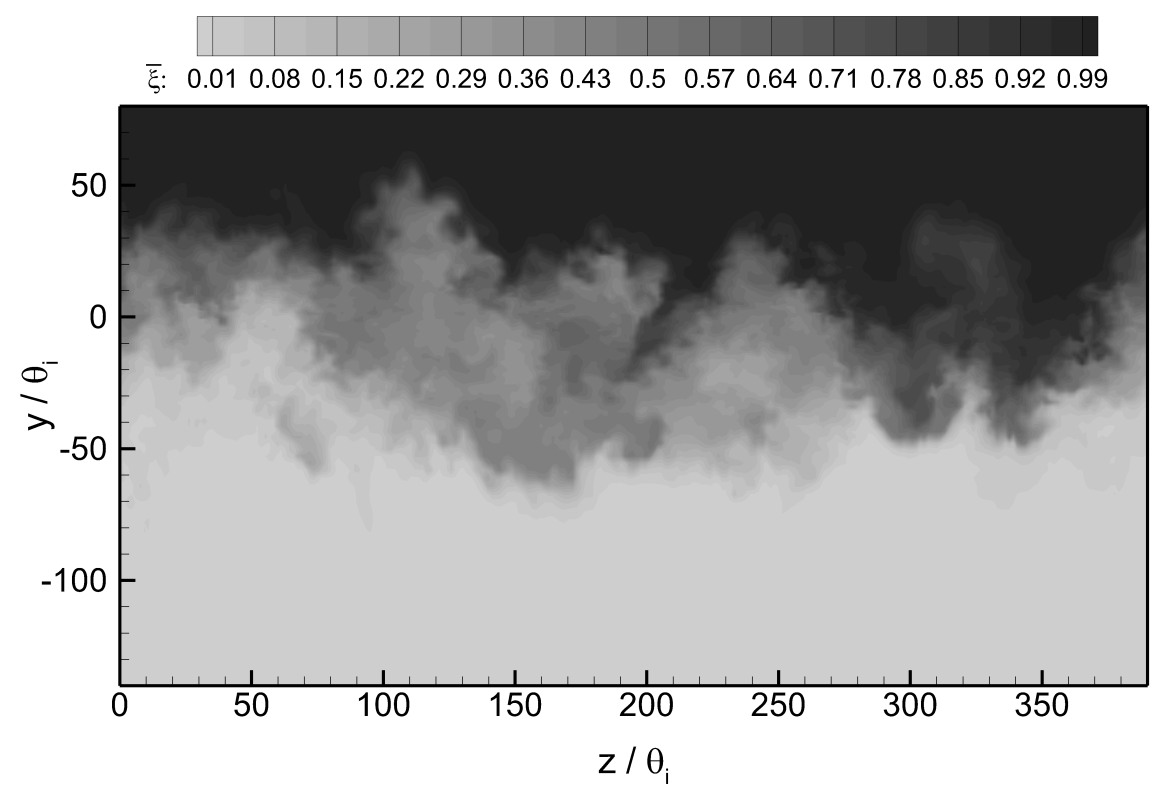

(a) Case LWN.

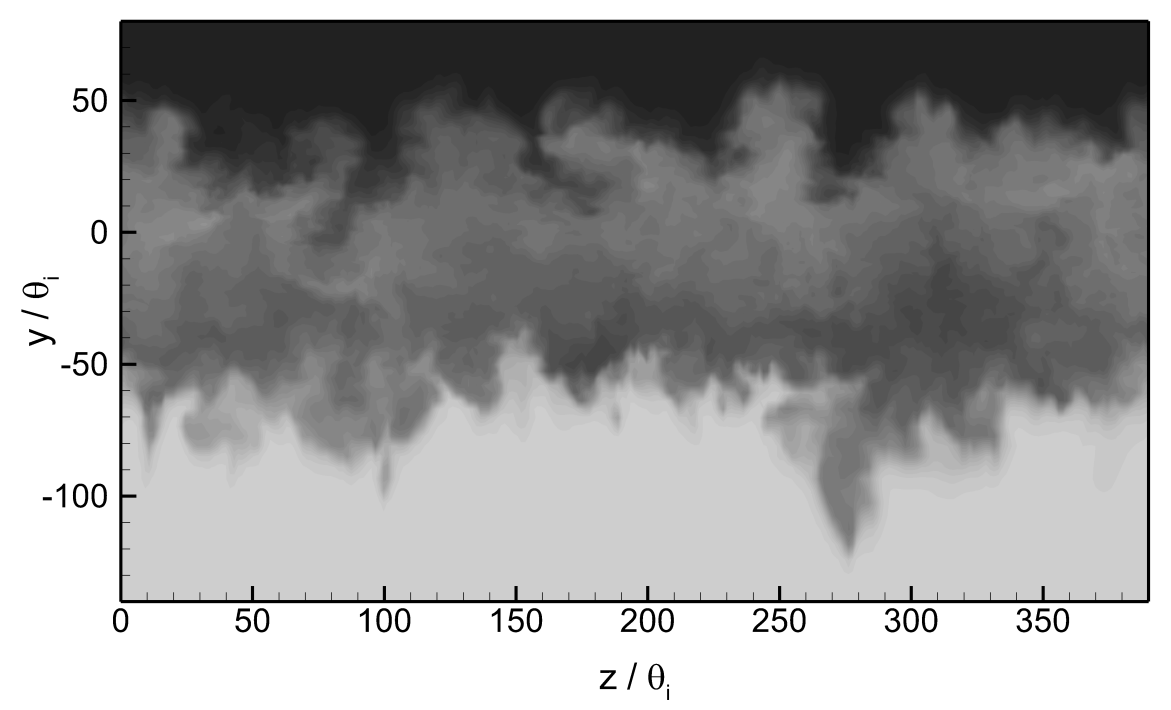

(b) Case LCF.

Figure 11: $y-z$ scalar distribution through the core of the vortices shown in Figure 10. (a) Case LWN, (b) Case LCF. 


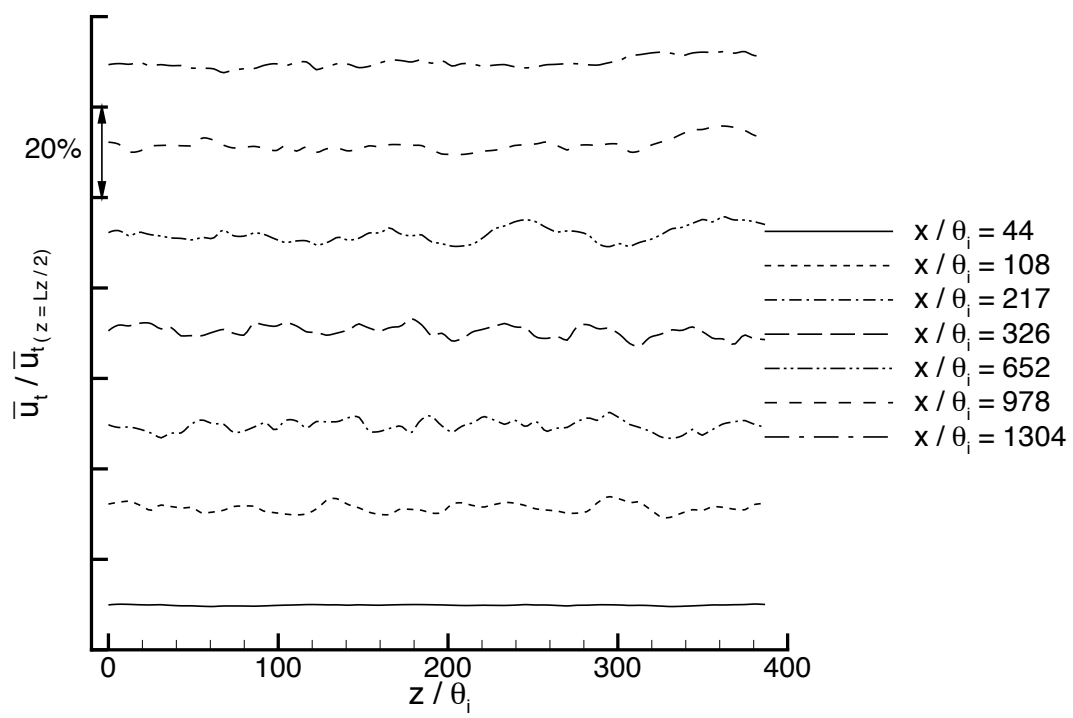

(a) Case LWN.

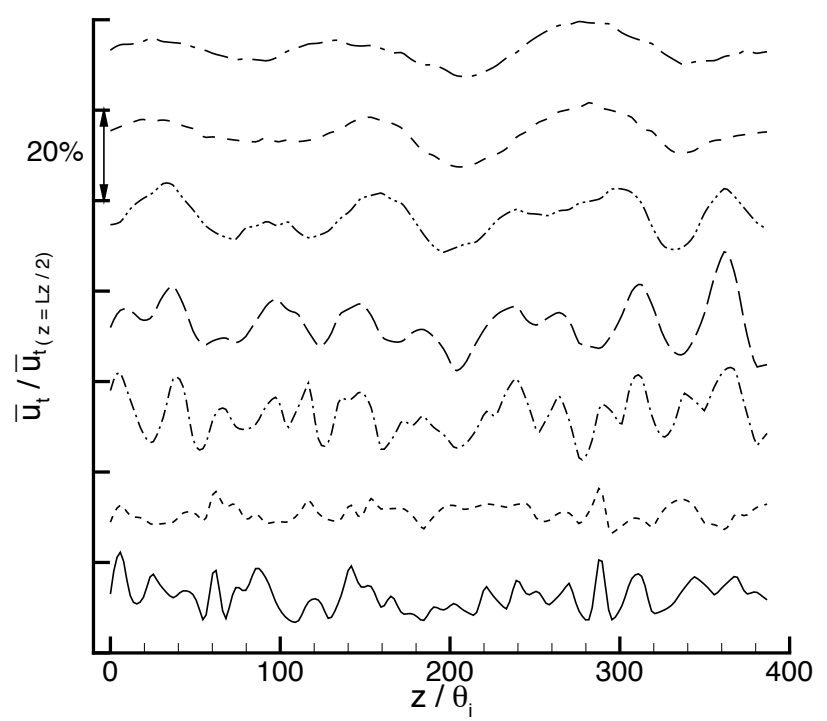

(b) Case LCF.

Figure 12: Spanwise variation of the mean streamwise velocity along the plane of the splitter plate $\left(y / \theta_{i}=0\right)$. (a) Case LWN, (b 45 Case LCF. 


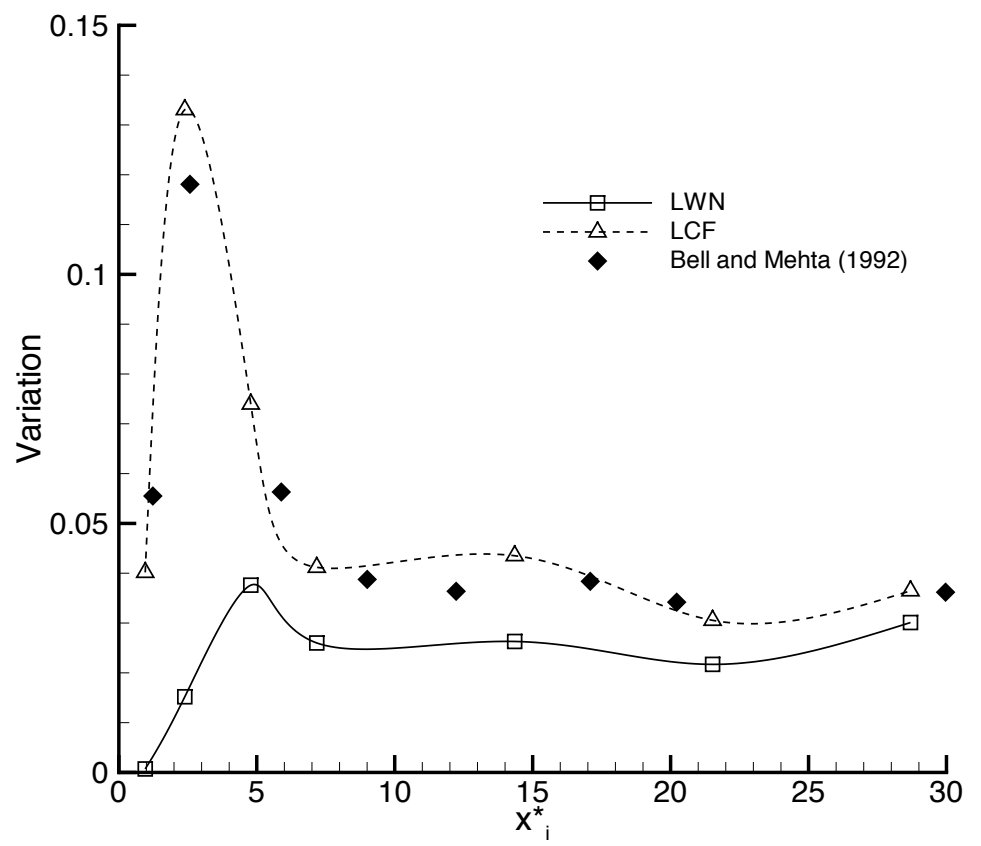

Figure 13: Spanwise variation of the mixing layer thickness. 

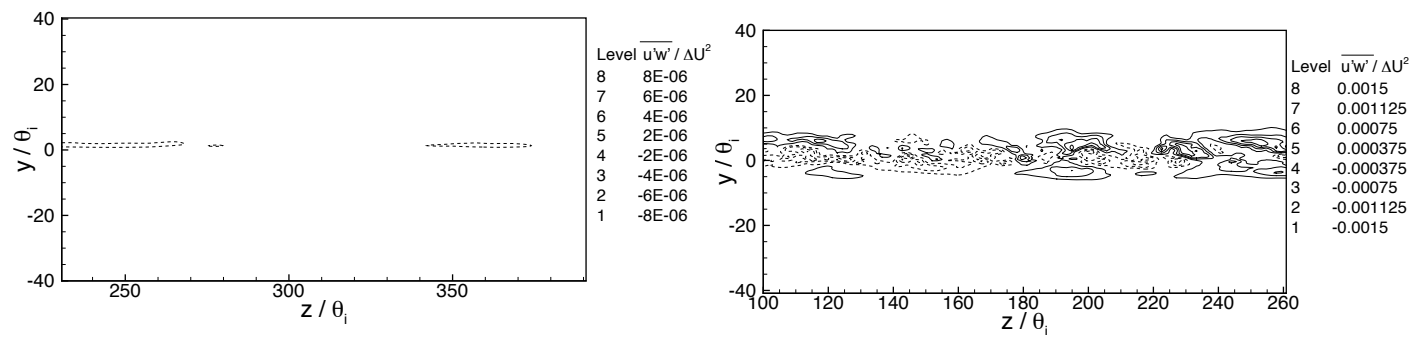

(a) $x / \theta_{i}=44$.

(b) $x / \theta_{i}=108$.
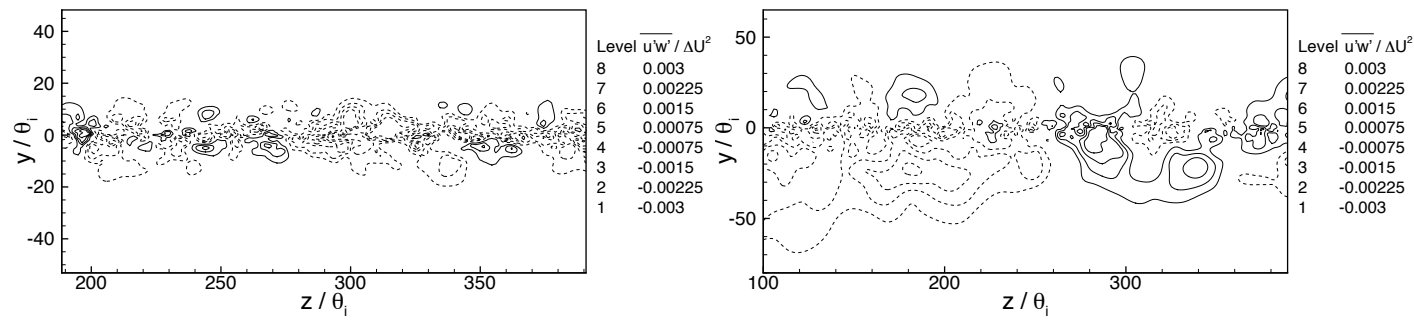

(c) $x / \theta_{i}=217$.

(d) $x / \theta_{i}=652$.

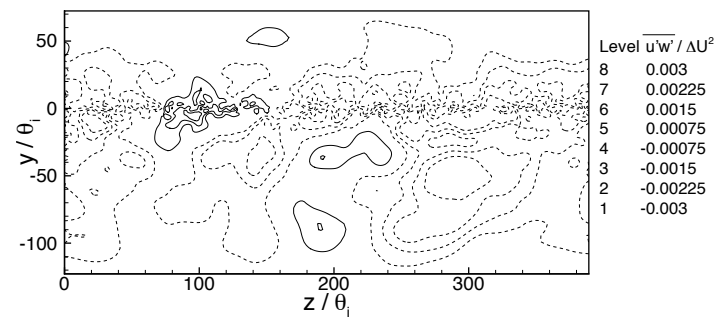

(e) $x / \theta_{i}=1304$.

Figure 14: Cross-plane secondary shear stress contours in Case LWN. Note the changes in axes extent and contour levels in each image. Dashed lines denote negative values. (a) $x / \theta_{i}=44$, (b) $x / \theta_{i}=108$, (c) $x / \theta_{i}=217$, (d) $x / \theta_{i}=652$, (e) $x / \theta_{i}=1304$. 

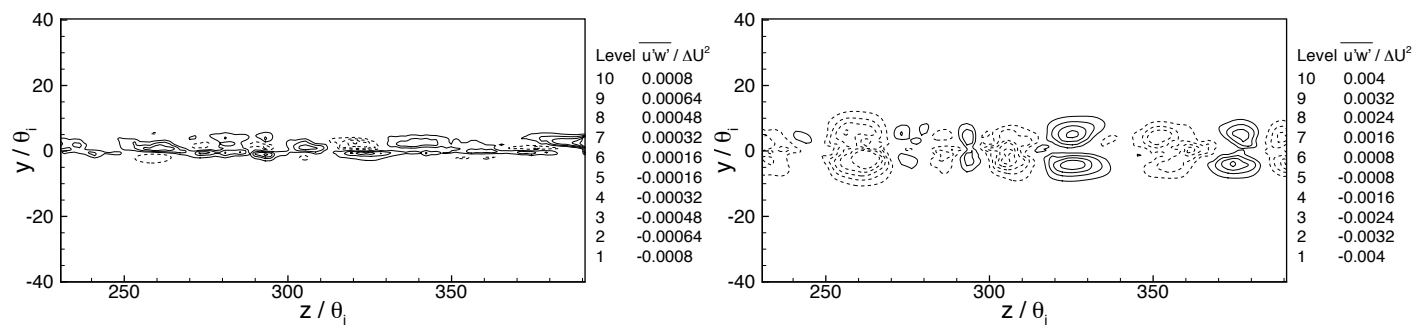

(a) $x / \theta_{i}=44$.

(b) $x / \theta_{i}=108$.
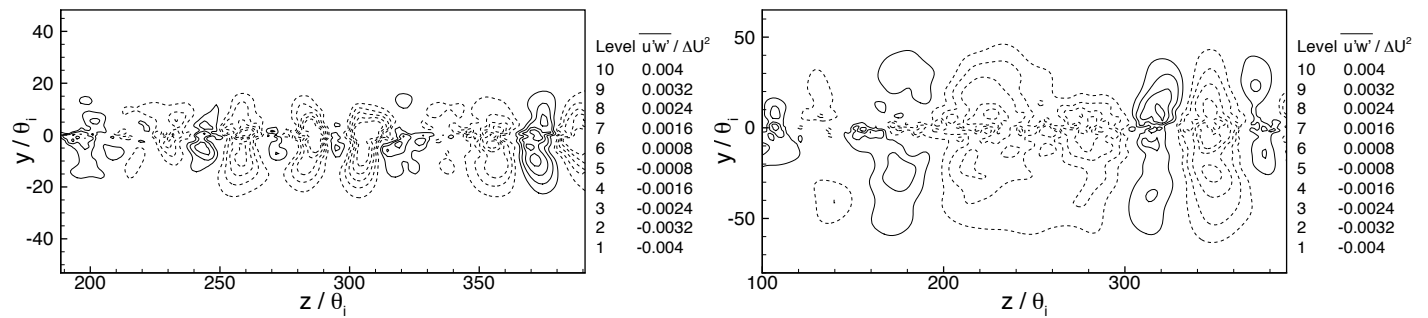

(c) $x / \theta_{i}=217$.

(d) $x / \theta_{i}=652$.

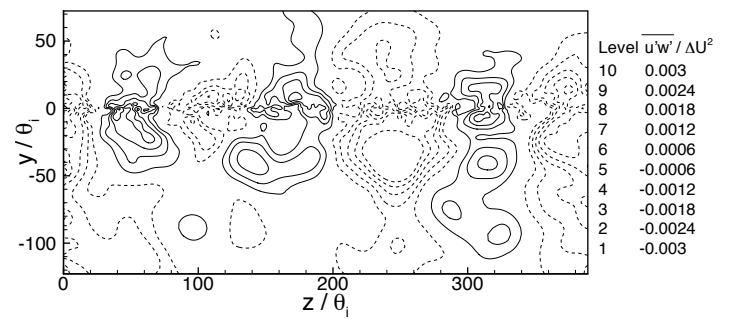

(e) $x / \theta_{i}=1304$.

Figure 15: Cross-plane secondary shear stress contours in Case LCF. Note the changes in axes extent and contour levels in each image. Dashed lines denote negative values. (a) $x / \theta_{i}=44$, (b) $x / \theta_{i}=108$, (c) $x / \theta_{i}=217$, (d) $x / \theta_{i}=652$, (e) $x / \theta_{i}=1304$. 